\title{
Characterisation of the Covalently-Bound Anionic Flavin Radical in Monoamine Oxidase A by Electron Paramagnetic Resonance
}

\begin{tabular}{|r|l|}
\hline Journal: & Journal of the American Chemical Society \\
\hline Manuscript ID: & ja076090q.R1 \\
\hline Danuscript Type: & Article \\
\hline Date Submitted by the Author: & 17-Oct-2007 \\
\hline Complete List of Authors: & $\begin{array}{l}\text { Kay, Christopher } \\
\text { El Mkami, Hassane } \\
\text { Molla, Gianluca } \\
\text { Pollegioni, Loredano } \\
\text { Ramsay, Rona }\end{array}$ \\
\hline
\end{tabular}

\section{SCHOLARONE ${ }^{\text {m }}$ \\ Manuscripts}




\title{
Characterisation of the Covalently Bound Anionic
}

\section{Flavin Radical in Monoamine Oxidase A by Electron}

\section{Paramagnetic Resonance}

\author{
Christopher W. M. Kay, ${ }^{*}$ Hassane El Mkami, ${ }^{\star}$ Gianluca Molla ${ }^{\S}$ Loredano Pollegioni ${ }^{\S}$ and Rona R. \\ Ramsay* \\ Department of Biology, University College London, Gower Street, London WC1E 6BT, UK \\ Centre for Biomolecular Sciences and Department of Physics and Astronomy, University of St. \\ Andrews, North Haugh, St. Andrews, Fife KY16 9ST, UK
}

Department of Biotechnology and Molecular Sciences, University of Insubria, 21100 Varese, Italy

Email c.kay@ucl.ac.uk, $\underline{\text { rrr@st-and.ac.uk }}$

RECEIVED DATE

TITLE RUNNING HEAD: The covalently bound flavin radical in Monoamine Oxidase A

University College London

$\ddagger$ University of St Andrews

$\S$ University of Insubria 
Abstract: It was recently suggested that partially reduced monoamine oxidase (MAO) A contains an equilibrium mixture of an anionic flavin radical and a tyrosyl radical (Rigby et al. J. Biol. Chem. 280 (2005) 4627-4632). These observations formed the basis for a revised radical mechanism for MAO. In contrast, an earlier study of MAO B only found evidence for an anionic flavin radical (DeRose et al. Biochemistry 35 (1996) 11085-11091). To resolve the discrepancy, we have performed continuous-wave electron paramagnetic resonance at $94 \mathrm{GHz}$ (W-band) on the radical form of MAO A. A comparison with D-amino acid oxidase (DAAO) demonstrates that both enzymes only contain anionic flavin radicals. Pulsed electron-nuclear double resonance spectra of the two enzymes recorded at $9 \mathrm{GHz}(\mathrm{X}-$ band) reveal distinct hyperfine coupling patterns for the two flavins. Density Functional Theory calculations show that these differences can be understood in terms of the difference at C8 $\alpha$ of the isoalloxazine ring. DAAO contains a non-covalently bound flavin whereas MAO A contains a flavin covalently bound to a cysteinyl residue at $\mathrm{C} 8 \alpha$. The similar electronic structures and hydrophobic environments of MAO and DAAO, and the similar structural motifs of their substrates suggest that a direct hydride transfer catalytic mechanism established for DAAO (Umhau et al. Proc. Natl. Acad. Sci. USA 97 (2000) 12463-12468) should be considered for MAO.

KEYWORDS. Monoamine oxidase, flavin radical, electron paramagnetic resonance, electron-nuclear double resonance, density functional theory 


\section{Introduction}

Flavoproteins are common in all living organisms and carry out a wide range of essential tasks from electron transport to DNA repair. ${ }^{1,2}$ The prosthetic groups are flavin mononucleotide (FMN) ${ }^{1}$ or flavin adenine dinucleotide (FAD). The redox-active part of these molecules is a 7,8-dimethyl isoalloxazine moiety substituted at N(10) (Figure 1). In vivo, flavins are found in three oxidation states: fully reduced, radical and fully oxidized, with varying degrees of protonation. Hence, they can donate or accept either one electron or a hydride ion, and thus can couple redox processes between one electron and two electron donors and acceptors. The functionalization required to enable the catalysis of a specific process is regulated by the apoprotein to which the flavin cofactor is bound. ${ }^{2}$ Hydrogen bonding at N1, $\mathrm{O} 2, \mathrm{~N} 3 \mathrm{H}, \mathrm{O} 4$ and $\mathrm{N} 5$ of the isoalloxazine ring changes the redox properties of the flavin, as does a covalent linkage to an amino acid residue in the protein. Typically, the sulfur of a cysteine or one nitrogen of a histidine forms a bond to either $\mathrm{C} 6$ or $\mathrm{C} 8 \alpha$.

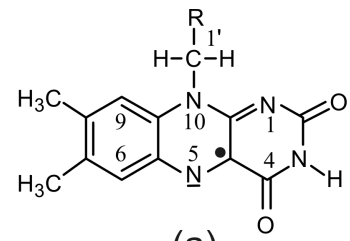

(a)

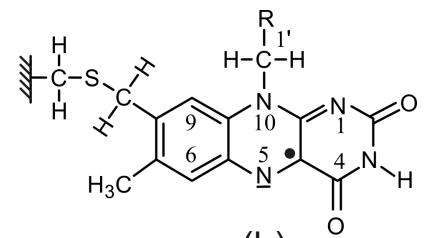

(b)

Figure 1. Molecular structures and IUPAC numbering scheme of anionic flavin radicals. (a) Noncovalently bound in DAAO and (b) covalently bound to cysteine at $\mathrm{C} 8 \alpha$ of the isolloxazine moiety in MAO.

In monoamine oxidase (MAO) $\mathrm{A}^{3}$ and the related enzyme, MAO B, the flavin is covalently linked to the protein by a cysteine residue bound at $\mathrm{C} 8 \alpha^{4}$ (Figure 1). MAO A and B are important pharmaceutical targets as they catalyze the oxidative deamination of neurotransmitters and xenobiotic amines, such as dopamine..$^{5,6}$

Despite intense research in many laboratories the mechanism of substrate oxidation in MAO remains to be clarified, with several alternatives having been proposed ${ }^{5,7}$ including a concerted nucleophilic 
mechanism ${ }^{8}$ and a single electron transfer (SET) mechanism. ${ }^{9}$ In the latter, the debate has been focused on the nature of a paramagnetic intermediate in these enzymes that might be sufficiently reactive to abstract an $\alpha$-hydrogen atom from the substrate. ${ }^{10-13}$

Yue and coworkers examined MAO B using continuous-wave electron paramagnetic resonance (cwEPR) and resonance Raman. ${ }^{10}$ The resonance Raman spectrum of the enzyme as isolated was virtually identical with its dithionite reduced form, and both resembled the anionic flavin radical found in glucose oxidase (GO). In dithionite reduced preparations, an EPR signal could be observed, although this had only $50 \%$ of the intensity expected when compared to the absorption spectrum. ${ }^{14,15}$ Hence, it was suggested that a second unidentified paramagnetic species was coupled to the flavin radical in MAO B. $^{10}$

Walker and Edmondson ${ }^{11}$ could not detect a flavin radical intermediate during turnover in MAO B, and with reference to ribonucleotide reductase (RNR), ${ }^{16}$ suggested that an amino acid radical would be a sufficiently oxidizing species to catalyse the reaction. However, they subsequently reported that they had found no evidence for a stable radical species in either MAO A or MAO B ${ }^{8}$.

Support for the idea that an amino acid radical is present in MAO A was presented by Rigby and coworkers who investigated the radical form of MAO A following anaerobic reduction with dithionite. ${ }^{13}$ The X-band cw-EPR and cw-ENDOR spectra were compared with those of GO, and the tyrosyl radical in Photoystem II. The authors proposed an equilibrium between an anionic flavin radical and a tyrosyl radical in MAO A. Furthermore, the unusually narrow EPR linewidth was used as evidence that the unpaired electron might be delocalized over a number of tyrosines (there are three in the active site), thus giving an exchange-narrowed spectrum. These observations formed the basis for a revised radical mechanism for MAO. ${ }^{7}$

DeRose and coworkers studied the radical form of MAO B, using cw-EPR and continuous-wave electron-nuclear double resonance (cw-ENDOR) at X-band $(9 \mathrm{GHz})$ and Q-band $(35 \mathrm{GHz}){ }^{12} \mathrm{In}$ concordance with Yue et al., ${ }^{10}$ a signal was detected in the resting state of the enzyme that could be ascribed to an anionic flavin radical. Photoreduction of the native enzyme in the presence of 
ethylenediaminetetraacetate, however, produced another radical with a distinct spectral signature that was tentatively assigned to a second flavin radical.

Thus, the EPR and ENDOR studies of MAO $\mathrm{A}^{13}$ and MAO $\mathrm{B}^{12}$ appear to give contradictory results, which given the highly similar structures of the two enzymes ${ }^{17,18}$ is surprising. Furthermore, although a tyrosyl radical has been observed in another flavoenzyme, (6-4)photolyase, it is only formed following oxidation by the photoexcited flavin, which is a far stronger oxidant than the ground state. ${ }^{19}$

In order to clarify the nature of the paramagnetic species in MAO A, we have performed cw-EPR at $95 \mathrm{GHz}$ (W-band), an approach that allows tyrosyl and flavin radicals to be differentiated by their characteristic g-tensors and spectral patterns. To corroborate our results we additionally present pulsed ENDOR spectra recorded at $9 \mathrm{GHz}$ (X-band). The results are broadly similar to those obtained for the resting state of MAO B. Furthermore, comparison with $D$-amino acid oxidase (DAAO) ${ }^{20}$ shows that the radicals in both enzymes have similar electronic structures. The comparison is relevant given that DAAO oxidizes $D$-alanine - which contains the same structural motif as substrates oxidized by MAO by direct hydride transfer to ${ }^{2} 5 .^{21-23}$ The mechanism of MAO is discussed in the light of these observations.

\section{Results}

W-band cw-EPR. In Figure 2, the W-band cw-EPR spectra of the anionic flavin radical in DAAO and MAO A are compared. Even at $94 \mathrm{GHz}$, the full anisotropy of the g-tensor is not fully resolved, but the similarity of the spectra gives us a high level of confidence that both are due to very similar species. 


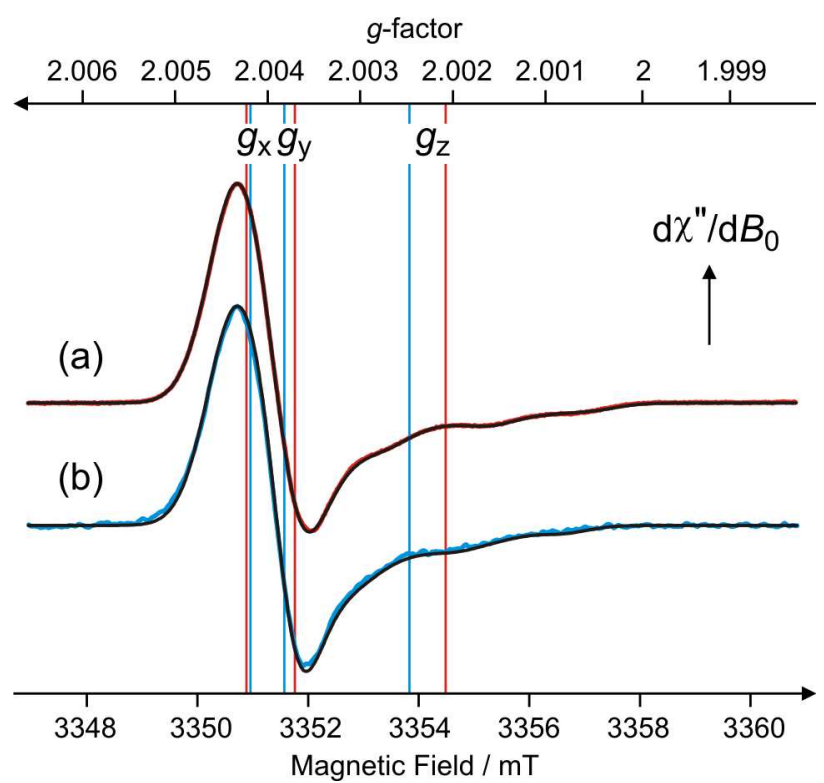

Figure 2. W-Band cw-EPR spectra (color) and simulations (black) of the anionic flavin radicals in (a) DAAO and (b) MAO A.

The broad linewidth and emerging structure is mainly due to the large hyperfine couplings hfcs of N5 and $\mathrm{N}_{10} 0^{24}$ and the g-anisotropy ${ }^{25}$ in anionic flavin radicals. By spectral simulation, it is possible to obtain reliable values of the principal values of the g-tensor and of the $A_{\|}$hfc values for N5 and N10. $A_{\perp}$ is set to zero for N5 and N10, and all other hfes are taken into account by including an anisotropic linewidth. For DAAO the principal values of the g-tensor are: $g_{x}=2.00427(5), g_{y}=2.00379(5)$ and $g_{z}=$ 2.00216(5) with $\mathrm{A}_{\|}=52(2) \mathrm{MHz}$ for N5 and $\mathrm{A}_{\|}=25(2) \mathrm{MHz}$ for N10. For MAO A: $\mathrm{g}_{\mathrm{x}}=2.00423(5), \mathrm{g}_{\mathrm{y}}$ $=2.00382(5)$ and $g_{z}=2.00247(5)$ with $\mathrm{A}_{\|}=48(2) \mathrm{MHz}$ for N5 and $\mathrm{A}_{\|}=29(2) \mathrm{MHz}$ for N10.

To date, there is only one report of a W-band EPR spectrum of an anionic flavin radical. ${ }^{25}$ In $\mathrm{Na}^{+}-$ Translocating NADH:quinone oxidoreductase $\left(\mathrm{Na}^{+}-\mathrm{NQR}\right)$ principal values of $\mathrm{g}_{\mathrm{x}}=2.00436(2), \mathrm{g}_{\mathrm{y}}=$ 2.00402(2) and $g_{z}=2.00228(2)$ have been reported. These values are uniformly 0.0001 larger than those we have determined, implying a small field calibration error. Axially symmetric hfes for N5 and N10 were also determined for the anionic flavin radical in $\mathrm{Na}^{+}-\mathrm{NQR}$, with $\mathrm{A}_{\|}=58 \mathrm{MHz}$ for $\mathrm{N} 5$ and $\mathrm{A}_{\|}=23$ MHz for N10 reported. 
The similarity of the W-band cw-EPR spectra of these enzymes is consistent with the presence of anionic flavin radicals. In MAO A, $g_{x}$ and $g_{y}$ are very similar to the values for the other enzymes, but $g_{z}$ shows a significant increase.

X-band pulsed-ENDOR. In Figure 3(a) the pulsed ENDOR spectrum of DAAO is presented, together with its first derivative (b). The latter is presented to ease comparison with the first derivative cw-ENDOR spectra of anionic flavin radicals found in the literature. The general features of the spectrum are similar to previously reported cw-ENDOR spectra for DAAO ${ }^{26}$ and other non-covalently

\section{Hyperfine Coupling / $\mathrm{MHz}$}

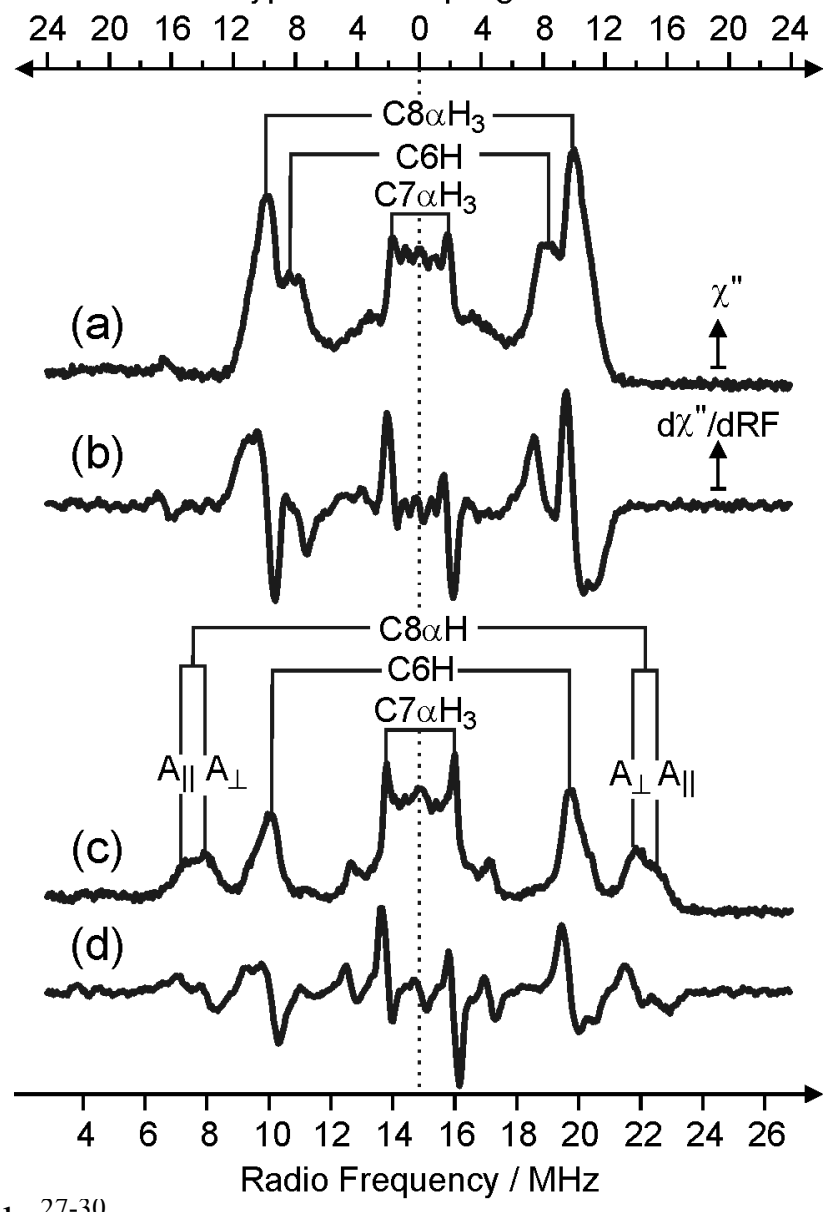

bound anionic flavin radicals. ${ }^{27-30}$ 
Figure 3. X-Band pulsed-ENDOR spectra of the anionic flavin radical in (a) DAAO and (c) MAO A. The first derivative spectra (b) and (d) were generated by pseudo-modulation ( $0.2 \mathrm{MHz}$ amplitude) of spectra (a) and (c), respectively.

In order to assist with assignment of the hfcs we have considered the high-resolution structure of DAAO (1COK) by Umhau and coworkers. ${ }^{22}$ The FAD was extracted from the structure, protons were added and optimized - for the anion radical state - using density functional theory, while holding the heavy atoms fixed. The molecular orbitals, the unpaired spin density distribution and hfcs were then calculated. The assignments made in the following are made with reference to the theoretical proton hfcs, which are presented in Table 1 . As found in previous work on flavins, ${ }^{30-32}$ the calculations are an excellent guide to the proton ENDOR frequencies, but also show that many of the transitions are anisotropic and overlapping. Hence, in the following we will only discuss those spectral features that can be assigned without resorting to specific isotope labeling.

- Insert Table 1 about here -

Hfcs in the range 5.7-12 MHz are assigned to $\mathrm{C} 6 \mathrm{H}, \mathrm{C} 8 \alpha \mathrm{H}_{3}$ and one of the two protons at $\mathrm{Cl}^{\prime}$. The three protons in a freely rotating methyl group coalesce to give a single axially symmetric hfc with small anisotropy. Hence, the intense feature with a hfc of $9.9 \mathrm{MHz}$ is assigned to $\mathrm{C} 8 \alpha \mathrm{H}_{3}$ and the hfc at 2.1 $\mathrm{MHz}$ is assigned to $\mathrm{C} \alpha \mathrm{H}_{3}$. Around $4 \mathrm{MHz}$ is another feature that is likely to be due to the other of the two $\beta$-protons bound at $\mathrm{Cl}^{\prime}$. Because the ribityl side chain is not free to rotate, the $\mathrm{C} 1^{\prime}-\mathrm{H}$ bonds make different dihedral angles, $\theta$, to the $\mathrm{p}_{\mathrm{z}}$ orbital of the N10 atom. The hfcs for $\beta$-protons have a $\cos ^{2} \theta$ dependence, and hence the proton with the smaller $\theta$ has a larger hfc and the one with a larger $\theta$ has a smaller H.

The pulsed ENDOR spectrum of MAO A is shown in Figure 3(c) together with its first derivative (d). Confirming our interpretation of the W-band cw-EPR, the spectra do not resemble the ENDOR spectra 
of the tyrosyl radicals in either photosystem $\mathrm{II}^{33}$ or RNR. ${ }^{34}$ Nevertheless, the spectral pattern, particularly for the larger hfcs, deviates significantly from that observed for DAAO. Specifically, a new feature with axial symmetry is present in the range $12-17 \mathrm{MHz}$, and the intensity in the $5.7-12 \mathrm{MHz}$ hfc region is diminished. As discussed above, the latter region is made up of protons at $\mathrm{C} 6, \mathrm{C} 8 \alpha$ and one of the two protons at $\mathrm{C1}^{\prime}$. The DFT calculations demonstrate that the hfes of $\mathrm{C} 6 \mathrm{H}$ and $\mathrm{C}^{\prime} \mathrm{H}$ do not differ significantly between DAAO and MAO A which only leaves the two protons at C8$\alpha$.

Inspection of several X-ray crystal structures of MAO reveals a narrow range of dihedral angles that the $\mathrm{C} 8 \alpha-\mathrm{S}$ bond makes with the isoalloxazine ring. ${ }^{17,18,35-39}$ This bond defines the dihedral angle, $\theta$, (and hence the $\mathrm{hfc}$ ) that the $\mathrm{C} 8 \alpha-\mathrm{H}$ bonds of the two protons make to the $\mathrm{p}_{\mathrm{z}}$ orbital of the $\mathrm{C} 8$ atom. For this analysis, we consider the high-resolution structure of MAO B (1OJA) by Binda and coworkers. ${ }^{35}$ The isoalloxazine ring moiety of the FAD and Cys397 (to which it is bound at C8 $\alpha$ ) were extracted from the structure. Protons were added, and geometry optimized - for the anion radical state - using DFT, while holding the heavy atoms fixed. The molecular orbitals, unpaired spin density distribution and hfcs were then calculated. The proton hfcs are presented in Table 1. With respect to the isoalloxazine ring, the $\mathrm{C} 8 \alpha-\mathrm{S}$ bond makes a dihedral angle of $59^{\circ}$, and the two protons bound at $\mathrm{C} 8 \alpha$ make dihedral angles of $8^{\circ}$ and $57^{\circ}$. Hence, the in-plane proton has a small hfc, while the out-of-plane proton has a large hfc. For the latter, the calculations predict an axially symmetric hfc with $\mathrm{A}_{\perp}=13$ and $\mathrm{A}_{\|}=16$ MHz. These are close to the values $-\mathrm{A}_{\perp}=13.8 \mathrm{MHz}$ and $\mathrm{A}_{\|}=15.4 \mathrm{MHz}-$ observed in the ENDOR spectrum.

The singly occupied molecular orbitals (SOMO) of the DAAO and MAO B flavin radicals are presented in Figure 4. Just as the hfcs of which they are a direct reflection are similar in the two flavin radicals, the SOMOs are rather similar. These are the same orbitals (minus the unpaired electron) that define the catalytic activity of the oxidized flavin. We have also used DFT to calculate the electronic structure of a truncated structure of the flavin in MAO B, in which we have removed the cysteinyl linkage in silico. This change hardly affects the SOMO, see Figure 4(b), or the hfcs, see Table 1, which 
is perhaps not surprising as it is a $\beta$-linkage relatively far removed from positions of high electron density at $\mathrm{C} 4 \mathrm{a}$ and $\mathrm{N} 5$, and thus only indirectly affects the $\pi$-system.

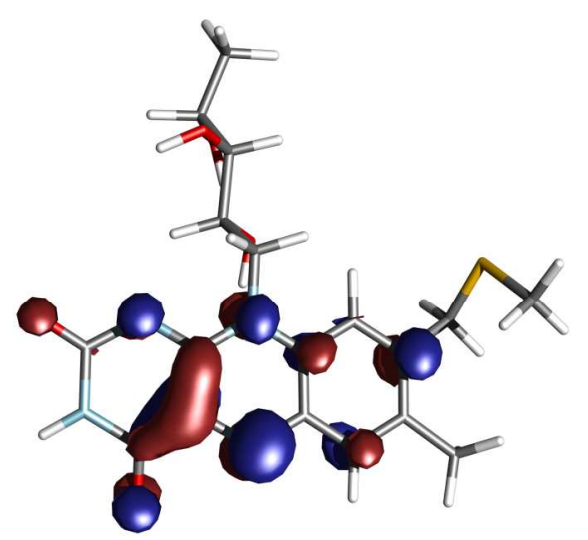

(a)

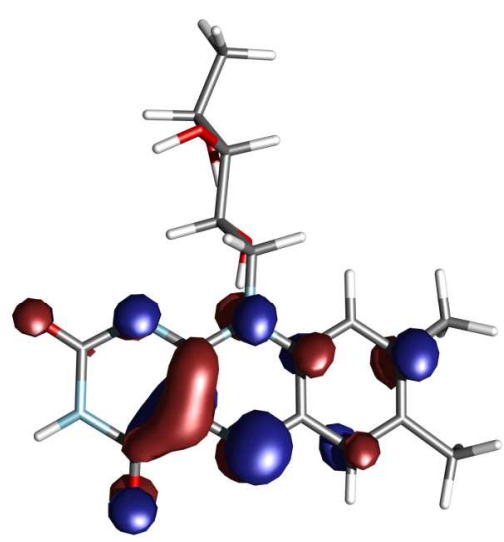

(b)

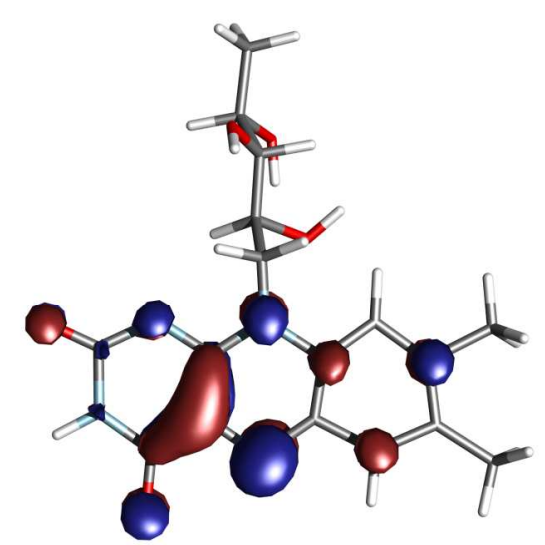

(c)

Figure 4. SOMOs calculated by DFT for the anionic flavin radicals. (a) MAO B, (b) MAO B without a covalent linkage to cysteinyl sulfur (c) DAAO. Blue and red indicate opposite signs of the wavefunction.

We note that our quantum chemical calculations do not include the protein environment which would be expected to fine tune the electronic properties of the flavin. ${ }^{31,}{ }^{32}$ Nevertheless the hfcs - the observables in the ENDOR experiment - of MAO and DAAO are very similar despite their different environments and the unusual butterfly shape of the flavin in MAO. Hence, if we accept the theory of orbital steering ${ }^{40}$ then having similar orbitals could be consistent with a similar mechanism.

\section{Discussion}

Characterization of the flavin radical. We conclude that there is no evidence for the presence of a tyrosyl radical in MAO A. The two well-characterized tyrosyl radicals, in photosystem II and 
ribonucleotide reductase (RNR), have completely different high-field cw-EPR spectra and g-tensor principal values. ${ }^{34,41,42}$ Rather, as previously shown for MAO B, ${ }^{12}$ the EPR spectrum is consistent with the presence of an anionic flavin radical.

The claim that a tyrosine radical is present in MAO A was also based on cw-ENDOR at X-band. ${ }^{13}$ Cw-ENDOR, using frequency modulation, is very useful for detecting narrow lines, such as the isotropic hfes of radicals in liquid solution. ${ }^{43}$ On the other hand, for protein-bound cofactors with large anisotropic hfcs, it is generally advantageous to use pulsed ENDOR so that complete tensorial patterns can be detected. ${ }^{30,44}$

Our results also reveal why the X-band cw-EPR spectrum of the covalently bound flavin radical in MAO is narrower than for non-covalently bound anionic flavin radicals, such as that in DAAO. Firstly, there is a reduction in the number of significant hfes present. In non-covalently bound anionic flavin radicals, the $\mathrm{C} 8 \alpha \mathrm{H}_{3}$ contributes three protons with a hfc of $\sim 10 \mathrm{MHz}$, whereas in MAO, the cysteinyllinkage reduces this number to two, of which only one has a significant hfc of $\sim 15 \mathrm{MHz}$. Secondly, the g-anisotropy is smaller. This likely to be caused by either enhanced spin-orbit coupling due to the sulfur atom or the butterfly structure of the flavin revealed by X-ray crystallography. ${ }^{17,18}$

Enzyme mechanism. X-ray crystallographic structures of MAO with various inhibitors or slow substrates covalently or non-covalently bound have shown that there is no amino acid in the active site that could act as an obvious initiator of catalysis. ${ }^{17,18,35-39}$ With this background, the mechanism by which the $\mathrm{C} \alpha-\mathrm{H}$ bond of the substrate is broken still disputed. $5,7,9,23,45$

One important point upon which there is general agreement, however, is that the substrate should arrive in the active site as an amine and not as an ammonium ion, which is its state in solution. ${ }^{46,47}$ Hence either in the hydrophobic environment of the binding site the $\mathrm{pK}_{\mathrm{a}}$ of the amine is lowered or the ammonium ion is deprotonated by a basic amino acid residue present in the entrance channel. ${ }^{48}$ That the unprotonated amine should be the substrate in MAO A has been inferred by investigations of the effect 
of the substituents in the aromatic ring of the substrate on the flavin reduction: a correlation of the $\mathrm{K}_{\mathrm{d}}$ values with the van der Waals volume of the para-substituents was observed. ${ }^{8}$

Our results have shown that following anaerobic titration there is no tyrosyl radical in MAO A, rather, as in MAO B, ${ }^{12}$ a covalently bound anionic flavin radical is generated. The tyrosyl radical formed the basis for one proposed reaction mechanism, ${ }^{13}$ which can now be excluded. In the following we discuss the alternatives.

Edmondson, Mattevi and coworkers have argued in favor of a nucleophilic mechanism, ${ }^{5,8}$ Figure 5(a). In this mechanism, the amine of the substrate acts as a nucleophile attacking the C4a position of the flavin, initially forming a covalent adduct. ${ }^{5}$ This leaves the flavin N5 negatively charged and with only two bonds and the substrate nitrogen atom positively charged with four bonds and present as a secondary ammonium ion. The proposal is that the strongly nucleophilic flavin N5 then abstracts a proton from $\mathrm{C} \alpha$, initially forming a negatively charged carbanion, adjacent to the positively charged nitrogen atom. This species then undergoes a rearrangement to form the imine product and reduced flavin. Fitzpatrick and coworkers have recently presented DFT calculations which are inconsistent with this type of mechanism. ${ }^{45}$ Furthermore, as noted by Silverman, ${ }^{9}$ this mechanism cannot readily explain the structure of the C4a covalent adduct formed with 2-phenylcyclopropylamine (PCPA) ${ }^{35}$ and the fact that MAO has no difficulty in oxidizing several sterically hindered secondary and tertiary amines. ${ }^{49}$ 
(a)

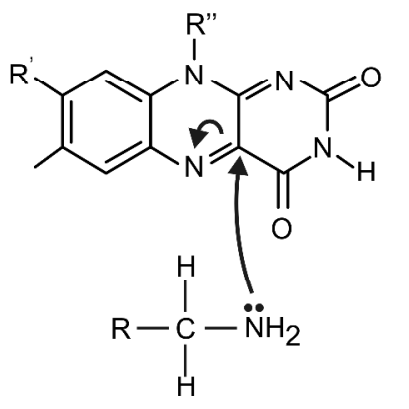

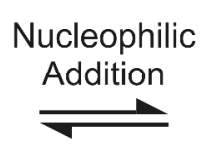

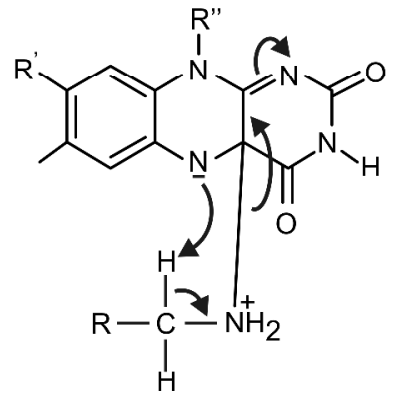<smiles></smiles>

(b)<smiles>[R]c1cc2c(cc1C)nc1c(=O)[nH]c(=O)nc-1n2[R7]</smiles><smiles>[R]CN</smiles>

(c)

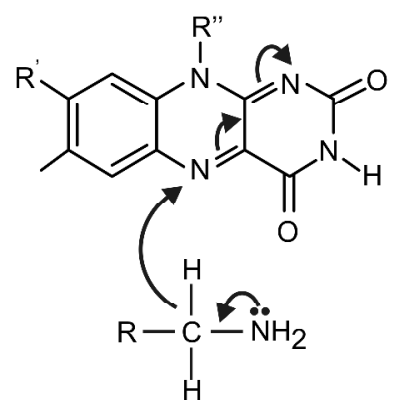<smiles>CCC</smiles><smiles>[R]c1cc2c(cc1C)Nc1c(nc(=O)[nH]c(=O)c1N)N2[R]</smiles><smiles>[18OH][TeH-]</smiles><smiles>[R]C[NH3+]</smiles>

Hydride Transfer

Figure 5. Proposed mechanisms for the oxidation of amines by MAO. (a) Nucleophilic mechanism, adapted from ref. ${ }^{8}$ (b) Single ET mechanism, adapted from ref. ${ }^{9}$ (c) Hydride transfer mechanism, based on the proposed mechanism of DAAO. ${ }^{22}$

Silverman and coworkers have performed extensive studies on cyclopropylamine analogs. ${ }^{9,}{ }^{50-53}$ For example, with isomers of PCPA, ring-opened covalent adducts were formed. Independent observations that amines may be oxidized chemically, ${ }^{54}$ electrochemically ${ }^{55}$ and photochemically ${ }^{56}$ were used as evidence that a single ET mechanism, Figure 5(b), might be active in MAO. ${ }^{9}$ One problem with basing an enzyme mechanism on this type of chemistry is that all these reactions are initiated by the input of energy, for example, a photon, whereas MAO oxidizes its substrates spontaneously. Furthermore, no 
paramagnetic intermediates have been observed during turnover by $\mathrm{EPR},{ }^{57}$ and the flavin radical could not be detected with stopped-flow optical spectroscopy. ${ }^{8,}{ }^{11}$ In order to rationalize the failure to observe a flavin radical, it was suggested that the radical intermediates must be very short lived, low in concentration, or spin paired. ${ }^{56}$ An alternative explanation is that the second ET is faster than the first. If this idea is pursued to its logical conclusion, then the reaction reduces to a hydride transfer, Figure 5(c). In a concerted rearrangement, the amine group of the substrate acts as a nucleophile, donating its lone pair to the $\mathrm{C} \alpha$, while the N1 of the flavin polarizes N5 which acts as an electrophile pulling the hydride ion off the substrate. The result is the direct formation of the imine and reduced flavin. The tendency of the amine to donate its lone pair to the $\mathrm{C} \alpha$ is illustrated by the observation that molecules such as $\mathrm{R}(\mathrm{R}) \mathrm{CX}-\mathrm{NH}_{2}$, (where $\mathrm{X}=\mathrm{F}, \mathrm{Cl}, \mathrm{Br}$ ) cannot be synthesized because the halide ions leave too easily. ${ }^{58}$ This pathway avoids the high energetic cost of forming an intermediate ionic radical pair ${ }^{45}$ and explains why no paramagnetic species are observed in MAO during turnover. The hydride ion would be expected to quantum mechanically tunnel through the transition state, a process that has been observed in MAO B. ${ }^{59}$ DFT calculations also predict that the reduced flavin is non-planar. ${ }^{45,60}$ Hence, enzymes, such as MAO that prebend the flavin will lower the barrier for hydride transfer.

In a crystal structure of trans,trans-farnesol with MAO B, ${ }^{38}$ the $\mathrm{C} \alpha$ is located $3.4 \AA$ from the flavin N5. Trans,trans-farnesol may be regarded as a biomimetic compound for amines with the difference being that the $\mathrm{NH}_{2}$ group is replaced by $\mathrm{OH}$, which renders it unreactive. This distance is comparable to that between the equivalent substrate/inhibitor $\mathrm{C} \alpha$ in amino acids and the flavin N5 in DAAO. ${ }^{22}$ Given a $\mathrm{C} \alpha-\mathrm{H}$ bond length of $1.1 \AA$, then the minimum distance between the proton and flavin N5 is $2.3 \AA$, which is a reasonable distance for hydride transfer.

A crystal structure of $p-\mathrm{NO}_{2}$-benzylamine with MAO B showed that the $\mathrm{C} \alpha$ is located at a very similar distance of $3.8 \AA$ from the flavin N5. ${ }^{39}$ Curiously, no clear electron density from the amine nitrogen was found, showing that this atom samples a range of conformations in the active site. Given that the $\mathrm{C} \alpha-\mathrm{N}$ bond direction determines the orientation of the protons bound at the $\mathrm{sp}^{3}$ hybridized $\mathrm{C} \alpha$, these must also 
be mobile. Hence random motion of the amine would allow (one of) these to obtain the correct position - with minimum distance - for efficient hydride transfer to the flavin N5.

Edmondson and coworkers have investigated the mechanism of MAO A and MAO B by kinetic isotope experiments on the oxidation of benzylamine derivatives. ${ }^{8,11,59}$ In all cases large kinetic isotope effects were observed demonstrating that the rate limiting step involved proton/deuteron transfer. This would also be consistent with a hydride transfer mechanism.

In MAO B, using steady-state kinetics, benzylamine had $k_{\text {cat }}=300 \mathrm{~min}^{-1}$, while $p$ - $\mathrm{NO}_{2}$-benzylamine had $k_{\text {cat }}=11.1 \mathrm{~min}^{-1.39}$ An electron withdrawing group such as $-\mathrm{NO}_{2}$ would aid the loss of the $\mathrm{C} \alpha$ proton, so in a nucleophilic mechanism (Figure 5a) the rate would be expected to increase, contrary to the experimental data. The reduction of rate is, however, consistent with a hydride transfer mechanism (Figure 5c). The effect is in the same direction, albeit diminished, with phenylethylamine derivatives. Phenylethylamine had $k_{\text {cat }}=228 \mathrm{~min}^{-1}$, while $p-\mathrm{NO}_{2}$-phenylethylamine had $k_{\mathrm{cat}}=111 \mathrm{~min}^{-1}$. The comparison demonstrates that when there is no conjugation then the effect of substituents will be diminished.

Experiments on MAO A with para-substituted benzylamine derivatives, however, show the opposite effect: the more electrophilic the substituent, then the larger was the rate, although unfortunately no data were given for $p-\mathrm{NO}_{2}$-benzylamine. Hence, these results were used to support a nucleophilic mechanism for the oxidation of para-substituted benzylamine derivatives by MAO A. ${ }^{8}$

This contradictory behavior is most unusual for enzymes that share $71 \%$ sequence identity and identical cofactors. Kinetic data on a limited number of substituted 1-methyl-4-phenyl-1,2,3,6tetrahydropyridine (MPTP) substrates for MAO A and MAO B, however, did give a consistent picture. ${ }^{49}$ Only minor differences were found between $k_{\text {cat }} / K_{\mathrm{m}}$ in $4^{\prime}$ substituted analogs, which were uniformly poor substrates. In contrast, the $2^{\prime}$ substituted analogs were far better substrates. The substitution at the $2^{\prime}$ position hinders the planarity required for conjugation of the phenyl ring with the tetrahydropyridine moiety. Given that phenyl rings are weakly electron withdrawing structures then the difference in reactivity between the $2^{\prime}$ and $4^{\prime}$ isomers implies that the presence of conjugated electron withdrawing 
groups decreases reactivity of these substrates with MAO A and MAO B. Hence, these results support a hydride mechanism.

A comparison with DAAO which oxidizes substrates with the same $\mathrm{R}_{1}\left(\mathrm{R}_{2}\right) \mathrm{CH}-\mathrm{NH}_{2}$ motif is also informative. Although LAAO which catalyses the same reaction on enantiomeric substrates is a better structural match for MAO, ${ }^{17,61}$ DAAO shares many salient features of the active site. ${ }^{22}$ As in MAO the amino group is expected to be unprotonated ${ }^{62-65}$ and X-ray crystallography has provided compelling evidence that the reaction occurs by direct hydride transfer and without the involvement of amino acid functional groups. $^{22}$

\section{Conclusion}

We have presented experiments that find no evidence for a tyrosyl radical in MAO A, rather a covalently bound anionic flavin radical was observed. We suggest that X-band cw-EPR spectroscopy is not always a reliable method to distinguish between different radicals rather high-field cw-EPR should be the method of choice.

We propose that a direct hydride transfer mechanism for the oxidation of aliphatic substrates should be considered for MAO. The key is that the deprotonated amine acts as a nucleophile. Its target is not the flavin $\mathrm{C} 4 \mathrm{a}$ as proposed in a nucleophilic mechanism, but the neighboring carbon on the substrate. The proposal has the merit that it is a simple, one step, reversible reaction that makes many of the convoluted steps in other proposals redundant. A hydride mechanism is consistent with the positioning of $p-\mathrm{NO}_{2}$-benzylamine and trans,trans-farnesol in the active site, and with the C4a adduct from 2-PCPA inactivation. With the exception of one set of experiments on MAO A with benzylamine analogs, a hydride transfer mechanism is consistent with observations of the reactions of MAO A and MAO B with different substrates and inhibitors reported to date. We expect that our arguments will have relevance to ongoing discussions of the mechanism of other enzymes that oxidize amines, such as trimethylamine dehydrogenase, sarcosine oxidase and dimethylglycine oxidase. 


\section{Materials and Methods}

Enzymes. MAO A (human liver form) expressed in S. cerevisiae was purified and stored at $-20^{\circ} \mathrm{C}$ in a solution of $50 \mathrm{mM}$ potassium phosphate, $\mathrm{pH} 7.2,0.8 \%$ n-octyl- $\beta$-D-glucopyranoside (Melford Laboratories Ltd, UK), $1.5 \mathrm{mM}$ dithiothreitol, $0.5 \mathrm{mM}$ D-amphetamine, and 50\% glycerol. ${ }^{3,} 66$ Before use, dithiothreitol, D-amphetamine, and glycerol were removed by gel filtration in a spin column of G50 Sephadex equilibrated with $50 \mathrm{mM}$ potassium phosphate, $\mathrm{pH} 7.2$, containing $0.05 \%$ Brij. The enzyme preparation was at least $98 \%$ pure on the basis of silver-stained SDS-PAGE analysis and was $97 \%$ active on the basis of flavin reduced by substrate compared to dithionite reduced enzyme.

Recombinant DAAO from the yeast Rhodotorula gracilis was expressed and purified from Escherichia coli BL21(DE3)pLysS cells by using the pT7-DAAO expression system ${ }^{67}$. The enzyme (in $50 \mathrm{mM}$ sodium pyrophosphate, $\mathrm{pH} 8.5,10 \%$ (v/v) glycerol, $0.3 \mathrm{mM}$ EDTA, and $0.5 \mathrm{mM} 2-$ mercaptoethanol) was judged homogeneous by SDS-PAGE electrophoresis and was fully active (specific activity of $120 \mathrm{U} / \mathrm{mg}$ protein on D-alanine as substrate).

One-electron reduction of MAO A (about $100 \mu \mathrm{M}$ ) with dithionite in an anaerobic cuvette was monitored in a Shimadzu UV-2101PC spectrophotometer. The radical samples were transferred anaerobically to EPR tubes and frozen in liquid nitrogen. The DAAO radical was prepared by anaerobic photoreduction in the presence of EDTA directly in the EPR tube, after which the samples were frozen in liquid nitrogen.

EPR instrumentation. W-band continuous-wave EPR spectra were recorded on two spectrometers: a custom-built spectrometer at St Andrews ${ }^{68}$ and a Bruker BioSpin Elexsys E680 spectrometer at the Free University Berlin both operating at $94 \mathrm{GHz}$. Conditions for all experiments were: temperature: $100 \mathrm{~K}$; microwave power: 50nW; modulation amplitude: $0.2 \mathrm{mT}$ (frequency: $100 \mathrm{kHz}$ ). A Li:LiF sample was 
used for g-factor calibration. Simulations were performed with the computer program SIMPOW6 obtained from Mark J. Nilges and the Illinois EPR research center (http://ierc.scs.uiuc.edu/nilges.html).

X-band pulsed ENDOR spectra were recorded using a pulsed EPR spectrometer (Bruker Elexsys E580) together with a DICE-ENDOR accessory including a radio-frequency amplifier (Amplifier Research 250A250A) and a dielectric-ring ENDOR resonator (Bruker EN4118X-MD-4-W1), which was immersed in a helium-gas flow cryostat (Oxford CF-935). All experiments were performed at $80 \mathrm{~K}$. For Davies-type ENDOR, a microwave pulse-sequence $\pi-t-\pi / 2-\tau-\pi$ using 64 and $128 \mathrm{~ns} \pi / 2-$ and $\pi$-pulses, respectively, and an RF pulse of $10 \mu$ s duration and starting $1 \mu$ s after the first microwave pulse were used. The separation times $t$ and $\tau$ between the microwave pulses were selected to be $13 \mu \mathrm{s}$ and $500 \mathrm{~ns}$, respectively. The entire pulse pattern was repeated with a frequency of $200 \mathrm{~Hz}$ so as to avoid saturation effects due to long electronic relaxation times.

Computation. In order to calculate electronic structures of the anionic flavin radicals, high-resolution $(1.2 \AA)$ X-ray structures were used for MAO B (10AJ) and DAAO (1COK). The structure of the flavin in MAO $\mathrm{A}$ is identical to that in MAO B. ${ }^{18}$ Protons were added as appropriate and energy optimized using DFT at the unrestricted B3LYP/6-311G(d,p) level of theory, as implemented in program package Gaussian 03. ${ }^{69}$ Hfcs for the anionic flavin radicals were calculated for the optimized structure at the same level of theory. Graphical representation of molecular orbitals was achieved using the Molden program package, ${ }^{70}$ followed by rendering with POV-Ray ${ }^{\mathrm{TM}}$. 
Acknowledgement. We are grateful to Dr Graham Smith (University of St Andrews) and Prof Robert Bittl (Free University Berlin) and for making available their W-band EPR spectrometers. CK thanks Dr Freidhelm Lendzian (Technical University Berlin) and Dr Christian Teutloff (Free University Berlin) for helpful discussions concerning the ENDOR spectra of tyrosyl radicals. This work was supported by the RCUK Basic Technology Programme through the HIPER project at St Andrews, and FAR grants to LP and GM. Access to the UCL Research Computing Services for DFT calculations is gratefully acknowledged. 


\section{REFERENCES}

(1) Massey, V. Biochem. Soc. Trans. 2000, 28, 283-296.

(2) Fraaije, M. W.; Mattevi, A. Trends. Biochem. Sci. 2000, 25, 126-132.

(3) Weyler, W.; Titlow, C. C.; Salach, J. I. Biochem. Biophys. Res. Comm. 1990, 173, 1205-1211.

(4) Kearney, E. B.; Salach, J. I.; Walker, W. H.; Seng, R. L.; Kenney, W.; Zeszotek, E.; Singer, T. P. Eur. J. Biochem. 1971, 24, 321-327.

(5) Edmondson, D. E.; Mattevi, A.; Binda, C.; Li, M.; Hubalek, F. Curr. Med. Chem. 2004, 11, $1983-$ 1993.

(6) Youdim, M. B.; Edmondson, D.; Tipton, K. F. Nat. Rev. Neurosci. 2006, 7, 295-309.

(7) Scrutton, N. S. Natural Product Reports 2004, 21, 722-730.

(8) Miller, J. R.; Edmondson, D. E. Biochemistry 1999, 38, 13670-13683.

(9) Silverman, R. B. Acc. Chem. Res. 1995, 28, 335-342.

(10) Yue, K. T.; Bhattacharyya, A. K.; Zhelyaskov, V. R.; Edmondson, D. E. Arch. Biochem. Biophys. 1993, 300, 178-185.

(11) Walker, M. C.; Edmondson, D. E. Biochemistry 1994, 33, 7088-7098.

(12) DeRose, V. J.; Woo, J. C.; Hawe, W. P.; Hoffman, B. M.; Silverman, R. B.; Yelekci, K.

Biochemistry 1996, 35, 11085-11091.

(13) Rigby, S. E.; Hynson, R. M.; Ramsay, R. R.; Munro, A. W.; Scrutton, N. S. J. Biol. Chem. 2005, 280, 4627-4631.

(14) Newton-Vinson, P.; Hubalek, F.; Edmondson, D. E. Protein Expr. Purif. 2000, 20, 334-345.

(15) Li, M.; Hubalek, F.; Newton-Vinson, P.; Edmondson, D. E. Protein Expr. Purif. 2002, 24, $152-162$.

(16) Stubbe, J. J. Biol. Chem. 1990, 265, 5329-5332.

(17) Binda, C.; Newton-Vinson, P.; Hubalek, F.; Edmondson, D. E.; Mattevi, A. Nat. Struct. Biol. 2002, 9, 22-26.

(18) De Colibus, L.; Li, M.; Binda, C.; Lustig, A.; Edmondson, D. E.; Mattevi, A. Proc. Natl. Acad. Sci. USA 2005, 102, 12684-12689.

(19) Weber, S.; Kay, C. W. M.; Mögling, H.; Möbius, K.; Hitomi, K.; Todo, T. Proc. Natl. Acad. Sci. USA 2002, 99, 1319-1322.

(20) Massey, V.; Ganther, H. Biochemistry 1965, 4, 1161-1173.

(21) Pollegioni, L.; Blodig, W.; Ghisla, S. J. Biol. Chem. 1997, 272, 4924-4934.

(22) Umhau, S.; Pollegioni, L.; Molla, G.; Diederichs, K.; Welte, W.; Pilone, M. S.; Ghisla, S. Proc. Natl. Acad. Sci. USA 2000, 97, 12463-12468.

(23) Fitzpatrick, P. F. Bioorg. Chem. 2004, 32, 125-139.

(24) Eriksson, L. E. G.; Ehrenberg, A. Biochim. Biophys. Acta 1973, 295, 57-66.

(25) Barquera, B.; Morgan, J. E.; Lukoyanov, D.; Scholes, C. P.; Gennis, R. B.; Nilges, M. J. J. Am. Chem. Soc. 2003, 125, 265-275.

(26) Kurreck, H.; Bretz, N. H.; Helle, N.; Henzel, N.; Weilbacher, E. J. Chem. Soc. Faraday Trans. 2 1988, 84, 3293-3306.

(27) Kurreck, H.; Bock, M.; Bretz, N.; Elsner, M.; Kraus, H.; Lubitz, W.; Muller, F.; Geissler, J.; Kroneck, P. M. H. J. Am. Chem. Soc. 1984, 106, 737-746.

(28) Edmondson, D. E. Biochem. Soc. Trans. 1985, 13, 593-600.

(29) Medina, M.; Vrielink, A.; Cammack, R. Eur. J. Biochem. 1994, 222, 941-947.

(30) Barquera, B.; Ramirez-Silva, L.; Morgan, J. E.; Nilges, M. J. J. Biol. Chem. 2006, 281, 364823691 .

(31) Garcia, J. I.; Medina, M.; Sancho, J.; Alonso, P. J.; Gomez-Moreno, C.; Mayoral, J. A.; Martinez, J. I. J. Phys. Chem. A 2002, 106, 4729-4735.

(32) Weber, S.; Möbius, K.; Richter, G.; Kay, C. W. M. J. Am. Chem. Soc. 2001, 123, 3790-3798.

(33) Rigby, S. E.; Nugent, J. H.; O'Malley, P. J. Biochemistry 1994, 33, 1734-1742. 
(34) van Dam, P. J.; Willems, J. P.; Schmidt, P. P.; Pötsch, S.; Barra, A. L.; Hagen, W. R.; Hoffman, B. M.; Andersson, K. K.; Gräslund, A. J. Am. Chem. Soc. 1998, 120, 5080-5085.

(35) Binda, C.; Li, M.; Hubalek, F.; Restelli, N.; Edmondson, D. E.; Mattevi, A. Proc. Natl. Acad. Sci. USA 2003, 100, 9750-9755.

(36) Ma, J. C.; Yoshimura, M.; Yamashita, E.; Nakagawa, A.; Ito, A.; Tsukihara, T. J. Mol. Biol. 2004, 338, 103-114.

(37) Binda, C.; Hubalek, F.; Li, M.; Herzig, Y.; Sterling, J.; Edmondson, D. E.; Mattevi, A. J. Med. Chem. 2004, 47, 1767-1774.

(38) Hubalek, F.; Binda, C.; Khalil, A.; Li, M.; Mattevi, A.; Castagnoli, N.; Edmondson, D. E. J. Biol. Chem. 2005, 280, 15761-15766.

(39) Li, M.; Binda, C.; Mattevi, A.; Edmondson, D. E. Biochemistry 2006, 45, 4775-4784.

(40) Mesecar, A. D.; Stoddard, B. L.; Koshland, D. E., Jr. Science 1997, 277, 202-206.

(41) Un, S.; Atta, M.; Fontecave, M.; Rutherford, A. W. J. Am. Chem. Soc. 1995, 117, 10713-10719.

(42) Bleifuss, G.; Kolberg, M.; Potsch, S.; Hofbauer, W.; Bittl, R.; Lubitz, W.; Gräslund, A.; Lassmann, G.; Lendzian, F. Biochemistry 2001, 40, 15362-15368.

(43) Kurreck, H.; Kirste, B.; Lubitz, W., Electron Nuclear Double Resonance Spectroscopy of Radicals in Solution. John Wiley and Sons: New York, 1988.

(44) Weber, S.; Kay, C. W. M.; Bacher, A.; Richter, G.; Bittl, R. ChemPhysChem 2005, 6, 292-299.

(45) Ralph, E. C.; Hirschi, J. S.; Anderson, M. A.; Cleland, W. W.; Singleton, D. A.; Fitzpatrick, P. F. Biochemistry 2007, 46, 7655-7664.

(46) McEwen, C. M., Jr.; Sasaki, G.; Lenz, W. R., Jr. J. Biol. Chem. 1968, 243, 5217-5225.

(47) McEwen, C. M., Jr.; Sasaki, G.; Jones, D. C. Biochemistry 1969, 8, 3952-3962.

(48) Jones, T. Z.; Balsa, D.; Unzeta, M.; Ramsay, R. R. J. Neural. Transm. 2007, 114, 707-712.

(49) Youngster, S. K.; McKeown, K. A.; Jin, Y. Z.; Ramsay, R. R.; Heikkila, R. E.; Singer, T. P. J. Neurochem. 1989, 53, 1837-1842.

(50) Silverman, R. B.; Hoffman, S. J. Biochem. Biophys. Res. Comm. 1981, 101, 1396-1401.

(51) Silverman, R. B. J. Biol. Chem. 1983, 258, 14766-14769.

(52) Silverman, R. B. Biochemistry 1984, 23, 5206-5213.

(53) Silverman, R. B.; Zieske, P. A. Biochemistry 1985, 24, 2128-2138.

(54) Hull, L. A.; Davis, G. T.; Rosenblatt, D. H. J. Am. Chem. Soc. 1969, 91, 6247-6250.

(55) Mann, C. K.; Barnes, K. K., In Electrochemical Reactions in Nonaqueous Systems, Mann, C. K., 'Ed.' Marcel Dekker: New York, 1970.

(56) Simpson, J. T.; Krantz, A.; Lewis, F. D.; Kokel, B. J. Am. Chem. Soc. 1982, 104, 7155-7161.

(57) Tan, A.; Glantz, M. D.; Piette, L. H.; Yasunobu, K. T. Biochem. Biophys. Res. Comm. 1983, 117, 517-523.

(58) Bailey, P. D.; Baker, S. R.; Boa, A. N.; Clayson, J.; Rosair, G. M. Tetrahedron Lett. 1998, 39, 7755.

(59) Jonsson, T.; Edmondson, D. E.; Klinman, J. P. Biochemistry 1994, 33, 14871-14878.

(60) Zheng, Y. J.; Ornstein, R. L. J. Am. Chem. Soc. 1996, 118, 9402-9408.

(61) Pawelek, P. D.; Cheah, J.; Coulombe, R.; Macheroux, P.; Ghisla, S.; Vrielink, A. EMBO J. 2000 , $19,4204-4215$.

(62) Fitzpatrick, P. F.; Massey, V. J. Biol. Chem. 1982, 257, 9958-9962.

(63) Denu, J. M.; Fitzpatrick, P. F. Biochemistry 1992, 31, 8207-8215.

(64) Kurtz, K. A.; Rishavy, M. A.; Cleland, W. W.; Fitzpatrick, P. F. J. Am. Chem. Soc. 2000, 122, 12896-12897.

(65) Harris, C. M.; Pollegioni, L.; Ghisla, S. Eur. J. Biochem. 2001, 268, 5504-5520.

(66) Weyler, W.; Salach, J. I. J. Biol. Chem. 1985, 260, 13199-13207.

(67) Molla, G.; Vegezzi, C.; Pilone, M. S.; Pollegioni, L. Protein Expr. Purif. 1998, 14, 289-294.

(68) Smith, G. M.; Lesurf, J. C. G.; Mitchell, R. H.; Riedi, P. C. Rev. Sci. Instrum. 1998, 69, 3924-3937.

(69) Gaussian 03, version Revision B.04; Gaussian, Inc: Pittsburgh PA, 2003.

(70) Schaftenaar, G.; Noordik, J. H. J. Comput. Aided Mol. Des. 2000, 14, 123-134. 


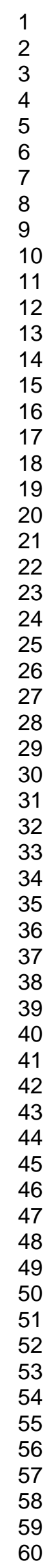


Table 1. Theoretical hfes for the protons of anionic flavin radicals in MAO B and DAAO calculated using DFT. $\mathrm{A}_{\text {iso }}$ is the isotropic hfc and $\mathrm{A}_{\text {dip }}$ is the dipolar component. All values are given in $\mathrm{MHz}$.

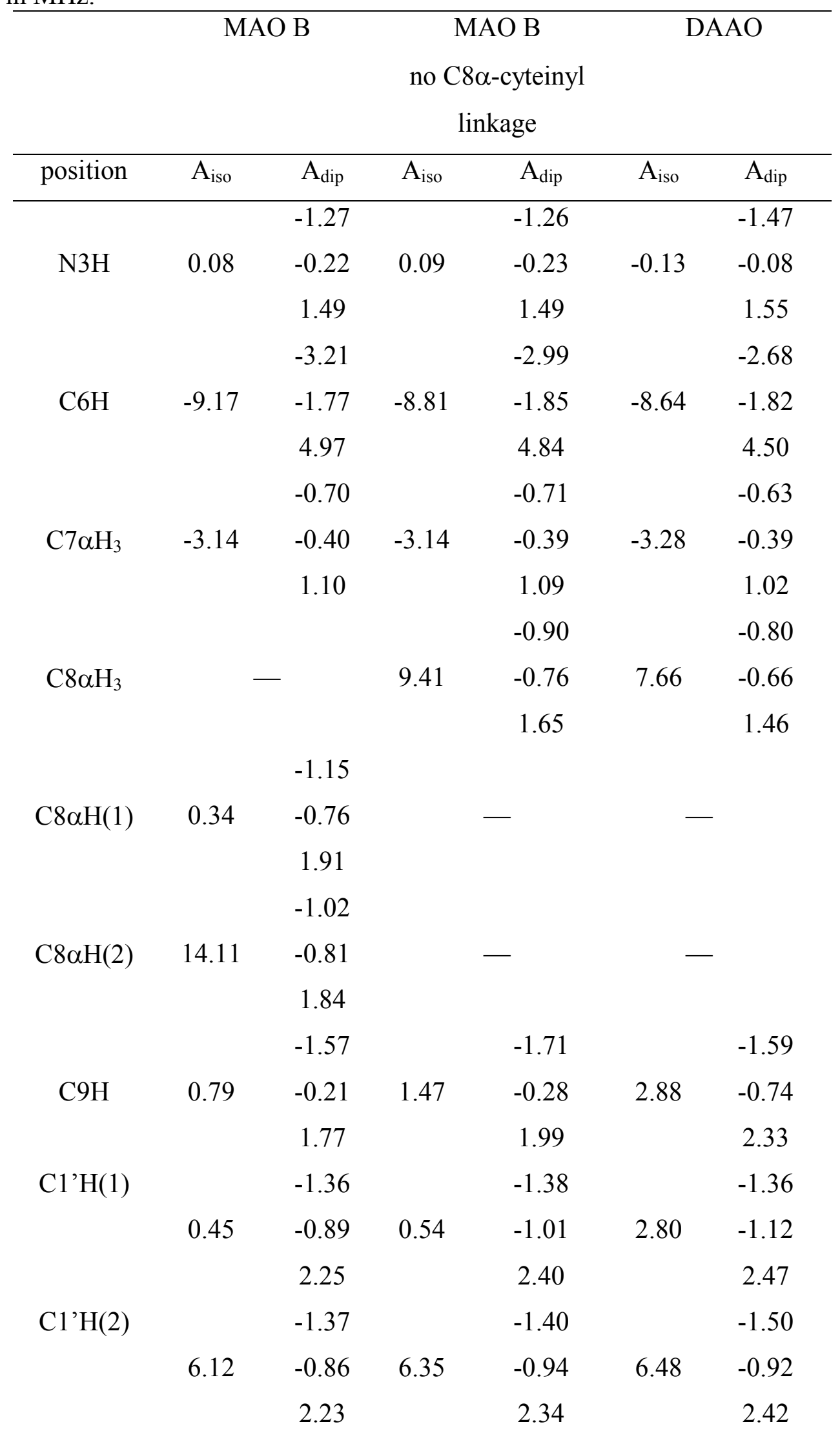




\section{SYNOPSIS TOC}

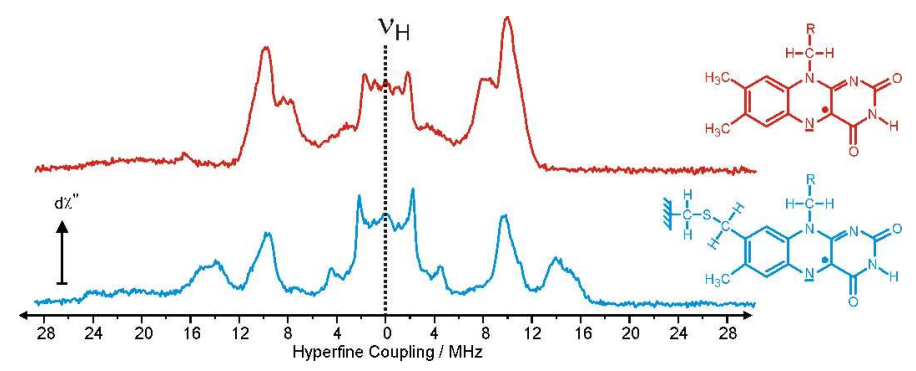

14

15

16

17

18

19

20

21

22

23

24

25

26

27

28

29

30

31

32

33

34

35

36

37

38

39

40

41

42

43

44

45

46

47

48

49

50

51

52

53

54

55

56

57

58

59

60 